\title{
Caracterização sociodemográfica e de consumo de substâncias psicoativas em usuários acompanhados em comunidade terapêutica
}

\author{
Sociodemographic characterization and consumption of psychoactive substances in users \\ monitored in the therapeutic community
}

Caracterización sociodemográfica y consumo de sustancias psicoactivas en usuarios monitoreados en la comunidad terapéutica

Tayla Quéren dos Santos Basso ORCID: https://orcid.org/0000-0002-1437-1641 Universidade Federal de Mato Grosso, Brasil E-mail: tayla-queren@live.com

Elias Marcelino da Rocha ORCID: https://orcid.org/0000-0002-0086-8286 Universidade Federal de Mato Grosso, Brasil E-mail: eliasufmt@yahoo.com.br

Vagner Ferreira do Nascimento ORCID: https://orcid.org/0000-0002-3355-163X Universidade Estadual de Mato Grosso, Brasil E-mail:vagnerschon@hotmail.com

Suzicléia Elizabete de Jesus Franco

ORCID: https://orcid.org/0000-0002-2288-9117

Universidade Federal de Mato Grosso Brasil E-mail: suzicleia@hotmail.com

Adaene Alves Machado de Moura ORCID: https://orcid.org/0000-0001-7607-9841 Universidade de São Paulo Brasil E-mail: adaene_moura@usp.br

Liliane Santos da Silva

ORCID: https://orcid.org/0000-0001-8639-874X Universidade de São Paulo, Brasil E-mail: liliane_rodrigues23@hotmail.com

Alisséia Guimarães Lemes

ORCID: https://orcid.org/0000-0001-6155-6473 Universidade Federal de Mato Grosso, Brasil E-mail: alisseia@hotmail.com

\begin{abstract}
Resumo
Objetivo: identificar as características sociodemográficas e de consumo de substâncias psicoativas de usuários internos em comunidades terapêuticas no interior do Vale do Araguaia, Brasil. Metodologia: trata-se de uma pesquisa transversal, cujos dados foram coletados através de um questionário semiestruturado, aplicados a usuários residentes em três Comunidades Terapêuticas que acolhem pessoas em situação de toxicodependência, destinadas ao público masculino. Resultados: foram entrevistados 37 internos, entre 19 e 70 anos, com média de 35,5 anos, solteiros, pardos, sem renda mensal, que cursaram até o ensino fundamental e com religião. $\mathrm{O}$ início do consumo de drogas foi majoritariamente entre os 12 e 17 anos, prevalecendo como primeira droga de uso o álcool, sendo que os motivos que levaram ao uso foram por curiosidade e para acompanhar amigos. O histórico familiar de uso foi prevalente, assim como o poliuso dessas substâncias, destacando o álcool e o crack como as substâncias que os levaram a dependência. Os indivíduos apresentaram consequências físicas e mentais do uso prolongado. A maioria dos entrevistados apresentaram um consumo de drogas diário antes de iniciar o tratamento. A procura pelo tratamento foi de forma voluntária ou a pedido dos familiares. Houve histórico de múltiplas internações, com destaque em serviço de comunidade terapêutica. Conclusão: torna-se necessário o desenvolvimento de estratégias de promoção e recuperação da saúde dos usuários que já se encontram em uso problemático de drogas, bem como a necessidade que se desenvolva ações de prevenção cada vez mais precoce entre a comunidade.Incluir o resumo.
\end{abstract}

Palavras-chave: Dependência química; Perfil de saúde; Comunidade terapêutica.

\section{Abstract}

Objective: to identify the sociodemographic and psychoactive substance consumption characteristics of internal users in therapeutic communities in the interior of the Araguaia Valley, Brazil. Methodology: this is a cross-sectional 
survey, whose data were collected through a semi-structured questionnaire, applied to users residing in three Therapeutic Communities that welcome people in situations of drug addiction, aimed at the male audience. Results: 37 inmates were interviewed, between 19 and 70 years old, with an average of 35.5 years old, single, brown, with no monthly income, who attended elementary school and with religion. The beginning of drug use was mostly between 12 and 17 years old, with alcohol as the first drug to be used, and the reasons that led to the use were out of curiosity and to accompany friends. The family history of use was prevalent, as well as the polius of these substances, highlighting alcohol and crack as the substances that led them to dependence. The individuals had physical and mental consequences of prolonged use. Most respondents had a daily drug use before starting treatment. The search for treatment was voluntary or at the request of family members. There was a history of multiple hospitalizations, especially in the therapeutic community service. Conclusion: it is necessary to develop strategies to promote and recover the health of users who are already in problematic use of drugs, as well as the need to develop preventive actions at an earlier age among the community.

Keywords: Chemical dependency; Health profile; Therapeutic community.

\section{Resumen}

Objetivo: identificar las características sociodemográficas y de consumo de sustancias psicoactivas de usuarios internos en comunidades terapéuticas del interior del Valle de Araguaia, Brasil. Metodología: se trata de una encuesta transversal, cuyos datos fueron recogidos a través de un cuestionario semiestructurado, aplicado a usuarios residentes en tres Comunidades Terapéuticas que acogen a personas en situación de adicción a las drogas, dirigido al público masculino.Resultados: Se entrevistaron 37 internos, entre 19 y 70 años, con un promedio de 35,5 años, solteros, morenos, sin ingresos mensuales, que asistían a la escuela primaria y tenían religión. El inicio del consumo de drogas fue mayoritariamente entre $\operatorname{los} 12$ y los 17 años, siendo el alcohol la primera droga que se consumió, y los motivos que llevaron al consumo fueron la curiosidad y el acompañamiento de amigos. Predominó el antecedente familiar de consumo, así como el polio de estas sustancias, destacando el alcohol y el crack como las sustancias que los llevaron a la dependencia. Los individuos tuvieron consecuencias físicas y mentales por el uso prolongado. La mayoría de los encuestados consumía drogas a diario antes de iniciar el tratamiento. La búsqueda de tratamiento fue voluntaria o por solicitud de familiares. Existía antecedente de múltiples hospitalizaciones, especialmente en el servicio comunitario terapéutico. Conclusión: es necesario desarrollar estrategias para promover y recuperar la salud de los usuarios que ya se encuentran en uso problemático de drogas, así como la necesidad de desarrollar acciones preventivas a una edad más temprana en la comunidad.

Palabras clave: Dependencia química; Perfil de salud; Comunidad terapéutica.

\section{Introdução}

Quase sempre as pessoas que utilizam Substâncias Psicoativas (SPA) buscam obter algum tipo de benefício neste consumo, seja como forma de obter prazer ou em busca de alívio de dores físicas ou da alma (Santiago, 2001; Bearn \& O'Brien, 2015). No entanto, o consumo de risco pode repercutir de forma negativa na saúde física e mental do indivíduo, expondo-o a diversas situações potencialmente prejudiciais, como brigas, desajuste familiar, prejuízos cognitivos, comportamentos de risco (acidente de transito, sexo desprotegido, entre outros), doenças crônicas e morte precoce, além de desencadear dependência em uma ou mais substância (Silva et al., 2016).

A dependência química é um problema de saúde pública, sendo caracterizada como "transtorno relacionado a substâncias e transtornos aditivos”, descrito no Manual de Diagnóstico e Estatístico de Transtornos Mentais (DSM-V) e também categorizado na Classificação Estatística Internacional de Doenças e Problemas Relacionados com a Saúde (CID10). Esse adoecimento mental apresenta características patológicas crônicas, recorrente com bases biológicas, genéticas, ambientais e culturais (Silva et al., 2016). Ainda está presente na literatura como uma condição clínica desde o século XVIII, isso se deve ao fato da urbanização decorrente da revolução industrial, a qual possibilitou aos médicos observar os problemas de saúde consequentes do uso prejudicial de drogas (Lessa, 1998).

Em 1924, avaliava-se no mundo, em torno de 100 mil adictos de drogas (Lessa, 1998). Desde então o número de usuários em situação de dependência química vem seguindo padrões crescentes, fator influenciado pela ampla disponibilização e forte comercialização das drogas lícitas de maneira agressiva por empresas multinacionais através de campanhas de publicidade que tem como maior objetivo o público jovem (United Nations Office on Drugs and Crime. World Drug Report, 2020). 
A ampla disponibilidade das substâncias psicoativas, assim como a ampliação do tráfico internacional de drogas e o aperfeiçoamento das substâncias disponíveis através da criação de novas drogas com um efeito mais danoso para o organismo, podem trazer consequências irreversíveis em pouco tempo (UNODC, 2020).

Dados do Escritório das Nações Unidas sobre Drogas e Crime (UNODC) apontam mais de 35 milhões de pessoas com transtorno relacionado ao consumo de substâncias psicoativas no mundo em 2017, 42 milhões de anos de vida "saudavel" perdidas em consequência a invalidez e morte prematura, e 585 mil mortes devido o uso de drogas que poderiam ser evitadas através de intervenções efetivas (UNODC, 2020).

Resultados obtidos no III Levantamento Domiciliar Sobre o Uso de Drogas na População Brasileira, levando em consideração todas as faixas etárias e ambos os sexos, apontam dependências do álcool (1,5\%), tabaco $(3,2 \%)$ e maconha (0,3\%), entre a população entrevistada (Bastos, Vasconcellos, Boni, Reis e Coutinho, 2017).

Os indivíduos em situação de dependência química se encontram em uma situação de vulnerabilidade, por isso requer um tratamento especializado, levando em consideração as particularidades de cada situação, a depender da intensidade da dependência, da aceitação do cliente/paciente/usuário, da presença de comorbidades e do perfil sociodemográfico (Silva et al., 2016). Os tratamentos disponíveis vão desde ambulatoriais na Atenção Básica (AB), especializados nos Centros de Atenção Psicossocial Álcool e Drogas (CAPS AD) e nos grupos de ajuda mútua (Alcoólicos Anônimos, Narcóticos Anônimos). Em casos mais graves, existe a possibilidade de internação, seja ela voluntária, involuntária ou compulsória, que pode ocorrer em hospitais gerais, hospitais psiquiátricos, clínicas especializadas e em Comunidades Terapêuticas (CT) (Brasil, 2019).

As CT são instituições que visam a recuperação de pessoas em situação de dependência química através da abordagem terapêutica comportamental, onde os usuários são os maiores responsáveis pela sua recuperação (BRASIL, 2019). O método utilizado é a abstinência, com um sistema constituído por meio de normas, horários e atividades, atribuindo funções, direitos e responsabilidades (Brasil, 2019). Porém, com a capacidade de risco e agravo à saúde dos usuários, esse modelo ainda requer adequações, com a presença de profissionais da saúde em tempo integral, planejamento para reinserção no mercado de trabalho e melhores estratégias de psicoeducação (Brasil, 2019).

Diante da alta complexidade dessa problemática, a prevenção ao uso e a intervenção antes da instalação da dependência química podem ser consideradas as melhores formas de enfrentamento, tendo em vista seus danos e consequências. Assim conhecer o perfil desses indivíduos apontando o contexto social, econômico e a fase de instalação da dependência, contribuem para o desenvolvimento de estratégias de prevenção, assim como tratamentos adequados a pessoas com condição clínica instalada.

Acredita-se ainda, que ao apresentar dados quantitativos que definam o perfil dos usuários em tratamento em comunidades terapêuticas, pode contribuir para melhoria da assistência por parte dos profissionais de saúde, uma vez que se torna possível compreender suas características, ampliando a compreensão de quem são as pessoas que buscam tratamento nessas instituições. Sendo assim, o objetivo deste trabalho foi identificar as características sociodemográficas e de consumo de substâncias psicoativas de usuários internos em comunidades terapêuticas no interior do Vale do Araguaia, Brasil.

\section{Metodologia}

Trata-se de uma pesquisa transversal, descritiva com abordagem quantitativa. De acordo com Rouquayrol (1994) a pesquisa transversal é um estudo epidemiológico no qual fator e efeito são observados num mesmo momento histórico. Gil (2008) descreve que a pesquisa descritiva consiste em observar e descrever um fenômeno, apoiando-se em métodos de análise estatística qualificados de "estatística descritiva". Permite visualizar uma situação e muitas vezes classificar, categorizar as variáveis ou as observações. E a abordagem quantitativa visa quantificar os dados, por meio do agrupamento 
das respostas dos entrevistados, e quantificá-los (Pereira et al., 2018).

A pesquisa foi realizada em três comunidades terapêuticas masculinas, localizadas no interior do Vale do Araguaia, Brasil. Essa região possui quatro Comunidades Terapêuticas, sendo três masculinas e uma feminina. As CT selecionadas para este estudo são instituições similares em relação à proposta de acompanhamento, de natureza religiosa, protestante evangélica, com capacidade para até 30 homens com idade $\geq 18$ anos, em situação de dependência química, com a exigência de se manterem abstinentes.

No momento das entrevistas, as instituições contavam apenas com tutores/monitores, pois não foi verificado a presença de nenhum profissional de nível superior, seja da área da saúde, social ou humanas. Os pesquisadores foram informados pelos gestores das CT que toda a assistência à saúde tem sido realizada no CAPS AD ou nos serviços de saúde local (hospital, Unidade de Pronto Atendimento ou na Estratégia de Saúde da Família) referências para as CT. Neste serviço de saúde mental para usuários de SPA, tem-se estabelecido um tempo de tratamento de seis a nove meses, sendo à entrada de forma voluntária e custeada por meios filantrópicos ou particulares.

Para a coleta de dados, utilizou-se um questionário semiestruturado elaborado pelos pesquisadores, contendo questões que contemplaram variáveis sociodemográficas (idade, estado civil, cor, renda, escolaridade, religião) e as características que definem o uso de SPA (idade de início do consumo de drogas, motivos que levaram ao uso, familiar usuário de drogas, primeira droga que usou, drogas mais utilizadas, frequência do consumo antes de iniciar o tratamento e número de internações).

Para avaliação das características dos usuários de SPA, foram entrevistados 37 internos, que preencheram os critérios de inclusão estabelecidos, sendo: usuário residente em uma das comunidades terapêuticas selecionadas para o estudo no período entre outubro de 2015 a dezembro de 2016; idade $\geq 18$ anos. Os critérios de exclusão foram: usuários que não foram encontrados na CT no momento da coleta de dados, seja por motivo de não adesão ao tratamento, ou por saída para consulta médica, judicial ou para tratar de motivos particulares. Foi excluído um indivíduo por não aceitar participar da pesquisa.

As entrevistas foram realizadas nos períodos matutinos e vespertinos, entre os meses de junho a dezembro de 2016, a partir da autorização dos gestores e usuários de cada CT, posterior ao esclarecimento dos objetivos do estudo, sendo realizada em um local calmo e silencioso disponibilizado pela instituição, em busca de proporcionar um ambiente no qual os participantes se sentissem seguros para responder o instrumento e pudessem relatar sua opinião, sem interrupções ou anseios. A aplicação dos instrumentos teve a duração média de 30 minutos. Em cada CT, retornou-se uma vez por mês para coleta de dados durante o período estabelecido, com vistas a atingir o máximo de usuários que davam entrada nas instituições.

Após a transcrição dos dados para um banco de dados da versão 3.5.2 do programa Epiinfo, foram realizados análises do tipo descritivo, e apresentados em forma de tabela e gráficos.

Foram respeitados todos os aspectos éticos em pesquisa com seres humanos de acordo com a Resolução 466/2012 do Conselho Nacional de Saúde, iniciando a investigação somente após aprovação do Comitê de Ética da UFMT/CUA, sob CAAE: 18394713.0 .0000 .5587 e parecer $\mathrm{n}^{\circ}$ 515/705. Todos participantes do estudo assinaram o Termo de Consentimento Livre e Esclarecido (TCLE).

\section{Resultados}

A pesquisa contou com a participação de 37 internos, do sexo masculino, com idade média de 35,5 anos, sendo a mínima de 19 anos e máxima de 70 anos. Na Tabela 1 é possível observar as características sociodemográficas dos residentes das comunidades terapêuticas participantes do estudo. 
Research, Society and Development, v. 10, n. 1, e22110111677, 2021

(CC BY 4.0) | ISSN 2525-3409 | DOI: http://dx.doi.org/10.33448/rsd-v10i1.11677

Tabela 1 - Distribuição das características sociodemográficas dos residentes das comunidades terapêuticas. Junho a dezembro de 2016. Região do Vale do Araguaia, Brasil, (n=37).

\begin{tabular}{|c|c|c|c|}
\hline Variáveis & Descrição & Frequência & $\%$ \\
\hline \multirow[t]{4}{*}{ Idade por faixa etária } & 19 a 29 anos & 11 & 30 \\
\hline & 30 a 40 anos & 17 & 46 \\
\hline & 41 a 51 anos & 08 & 21 \\
\hline & $\geq 52$ anos & 01 & 03 \\
\hline \multirow[t]{3}{*}{ Estado civil } & Solteiro & 31 & 84 \\
\hline & União estável & 04 & 11 \\
\hline & Divorciado & 02 & 05 \\
\hline \multirow[t]{3}{*}{ Cor da pele } & Pardo & 26 & 70 \\
\hline & Branco & 07 & 19 \\
\hline & Negro & 04 & 11 \\
\hline \multirow[t]{4}{*}{ Renda mensal } & Sem renda declarada & 19 & 51 \\
\hline & <01 salário mínimo* & 01 & 03 \\
\hline & 01 a 03 salários mínimo & 16 & 43 \\
\hline & >3 salários mínimo & 01 & 03 \\
\hline \multirow[t]{4}{*}{ Escolaridade } & Analfabeto & 01 & 03 \\
\hline & Ensino fundamental & 22 & 59 \\
\hline & Ensino médio & 13 & 35 \\
\hline & Ensino superior & 01 & 03 \\
\hline \multirow[t]{4}{*}{ Religião } & Evangélico & 26 & 70 \\
\hline & Católico & 07 & 19 \\
\hline & Espirita & 01 & 03 \\
\hline & Não possui & 03 & 08 \\
\hline
\end{tabular}

*Salário mínimo no período da pesquisa correspondia à 880,00 reais. Fonte: dados da pesquisa (2016).

Observa-se que 28 participantes tinham idade de 19 a 40 anos (76\%), predominando homens solteiros (84\%), pardos (70\%), sem renda mensal (51\%), que cursaram o ensino fundamental (59\%), com religião (92\%) e nesse aspecto, a maioria se autodeclaram evangélicos $(70 \%)$ (Tabela 1$)$.

Abaixo, a Tabela 2 apresenta as variáveis que definem as características do uso de substâncias psicoativas entre os participantes da pesquisa. 
Tabela 2 - Distribuição das características do consumo de Substâncias Psicoativas (SPA) entre os internos das comunidades terapêuticas. Junho a dezembro de 2016. Região do Vale do Araguaia, Brasil, (n=37).

\begin{tabular}{|c|c|c|c|}
\hline Variáveis & Descrição & Frequência & $\%$ \\
\hline & 9 a 11 anos & 08 & 22 \\
\hline Idade de início do & 12 a 14 anos & 14 & 38 \\
\hline \multirow[t]{2}{*}{ consumo de SPA } & 15 a 17 anos & 12 & 32 \\
\hline & 18 a 20 anos & 03 & 08 \\
\hline \multirow[t]{3}{*}{ Primeira droga de uso } & Álcool & 24 & 65 \\
\hline & Maconha & 07 & 19 \\
\hline & Tabaco & 06 & 16 \\
\hline \multirow{7}{*}{$\begin{array}{l}\text { Motivo do consumo de } \\
\text { drogas psicoativas* }\end{array}$} & Curiosidade & 25 & 68 \\
\hline & Acompanhar amigos & 25 & 68 \\
\hline & Influência familiar & 08 & 22 \\
\hline & Desilusão amorosa & 05 & 14 \\
\hline & Revolta pessoal & 04 & 11 \\
\hline & Influência de pares & 04 & 11 \\
\hline & Outros motivos & 05 & 14 \\
\hline Histórico familiar de & Sim & 27 & 73 \\
\hline \multirow[t]{2}{*}{ uso de SPA } & Não & 10 & 27 \\
\hline & Álcool & 15 & 41 \\
\hline \multirow{5}{*}{$\begin{array}{c}\text { Droga que levou a } \\
\text { dependência na } \\
\text { percepção do usuário }\end{array}$} & Crack & 12 & 32 \\
\hline & Pasta base & 03 & 08 \\
\hline & Maconha & 05 & 13 \\
\hline & Cocaína & 01 & 03 \\
\hline & Tabaco & 01 & 03 \\
\hline
\end{tabular}

*os internos puderam assinalar mais de uma opção. Fonte: dados da pesquisa (2016).

Verificou-se que o uso de SPA ocorreu de forma precoce, com início entre 9 a 20 anos, concentrando o uso de 9 a 14 anos (60\%). A droga de primeiro uso foi o álcool (65\%), seguido da maconha (19\%) e do tabaco (16\%), consumidas por curiosidade e para acompanhar os amigos com $68 \%$ cada, seguido da influência familiar (22\%). Entre os participantes do estudo, $73 \%$ afirmaram a existência de um familiar de primeiro grau com histórico do uso de SPA. E em relação a droga que o levou a dependência a maioria apontaram o álcool (41\%), seguido pelo crack (32\%) (Tabela 2).

Os participantes reconhecem que o consumo de substâncias psicoativas podem desencadear consequências em sua vida, assim como na vida de outras pessoas, conforme exposto na Tabela 3. 
Tabela 3 - Distribuição das consequências ocasionadas em decorrência do consumo de SPA entre os residentes das comunidades terapêuticas. Junho a dezembro de 2016. Região do Vale do Araguaia, Brasil, (n=37).

\begin{tabular}{|c|c|c|c|}
\hline Variáveis & Descrição & Frequência & $\%$ \\
\hline Prejuízos causados no usuário em decorrência & Sim & 24 & 65 \\
\hline do uso abusivo de SPA & Não & 13 & 35 \\
\hline Prejuízos causados a outras pessoas em & Sim & 18 & 49 \\
\hline decorrência do uso abusivo de SPA & Não & 19 & 51 \\
\hline Doença física adquirida após consumo abusivo & Sim & 18 & 49 \\
\hline de SPA & Não & 19 & 51 \\
\hline Doença mental adquirida após consumo & Sim & 21 & 57 \\
\hline abusivo de SPA & Não & 16 & 43 \\
\hline Histórico de tentativa de suicídio posterior ao & Sim & 11 & 30 \\
\hline consumo abusivo de SPA & Não & 26 & 70 \\
\hline
\end{tabular}

Fonte: Dados da pesquisa (2016).

Os dados acima revelam que 24 (65\%) usuários referiram ter algum tipo de prejuízo, enquanto que 18 (49\%) já causaram algum tipo de prejuízo a outras pessoas. Posterior ao início do consumo de drogas, 18 (49\%) internos desenvolveram algum tipo de doença física, com destaque para doenças das vias respiratórias, desnutrição e déficit neurológico. Já a doença mental esteve presente entre 21 (57\%) internos, com destaque para depressão e ansiedade. Entre os participantes do estudo, $11(30 \%)$ referiram tentativa de suicídio.

Os resultados apresentados na tabela abaixo descrevem às características relacionadas ao tratamento para dependência química, e o consumo antecedente a internação (Tabela 4). 
Tabela 4 - Distribuição das características do tratamento para dependência química dos residentes das comunidades terapêuticas. Junho a dezembro de 2016. Região do Vale do Araguaia, Brasil, (n=37).

\begin{tabular}{cccc}
\hline Variáveis & Descrição & Frequên & cia \\
\hline Drogas psicoativas & Álcool & 35 & 95 \\
utilizada anterior ao & Tabaco & 30 & 81 \\
tratamento* & Crack & 23 & 62 \\
& Maconha & 21 & 57 \\
\hline Frequência do consumo & Cocaína & 06 & 16 \\
de drogas anterior ao & 2 a 3 vezes ao dia & 65 \\
tratamento & $\geq 10$ vezes ao dia & 09 & 24 \\
& 2 a 3 vezes por semana & 02 & 5 \\
\hline para tratamento da & 1 vezes por semana & 1 & 3 \\
\hline dependência química & Terceira internação & 1 & 62 \\
\hline tratamento & A pedido da família & 23 & 30
\end{tabular}

* os internos puderam assinalar mais de uma opção. Fonte: Dados da pesquisa (2016).

Anterior a busca pelo tratamento atual, a droga de maior consumo descrita pelos participantes foi o álcool (95\%), seguido do tabaco $(81 \%)$, crack $(62 \%)$ e a maconha $(57 \%)$, consumidas diariamente entre $89 \%$ dos entrevistados, enquanto que para $11 \%$ o consumo foi semanal. Para $62 \%$ dos internos a entrada na CT ocorreu de forma voluntária, sendo que $51 \%$ dos entrevistados já buscaram por tratamento de duas a três vezes e 30\% estiveram quatro ou mais vezes internados para tratar a dependência química, o que demonstra uma recorrência na busca por tratamento (Tabela 4).

\section{Discussões}

Nesta pesquisa observou-se que os usuários apresentaram ser adultos jovens, predominando homens com idade entre 19 a 40 anos, semelhante ao apontado em estudo com usuários em tratamento em Comunidades Terapêuticas (Cosentino, Vianna, Souza e Perdonssini, 2017; Lemes, Rocha, Nascimento, Silva, Almeida e Villar, 2020) e em um CAPS AD (Silva, Torrezan, Costa, Garcia e Toledo, 2017) no Brasil. Apesar do início do uso de drogas ser precoce, a procura pelo tratamento tem sido atribuída a indivíduos na fase adulta, talvez essa seja a idade em que os anos de consumo tenha contribuído para os prejuízos à saúde física e mental dos usuários e estes reconheçam a necessidade de buscar por tratamento, por incentivo da família, ou até mesmo, por encaminhamento pela justiça (Capistrano et al., 2018).

Com relação ao estado civil, neste estudo predominou homens solteiros, assim como em outros estudos que avaliaram o perfil de usuários de drogas psicoativas em Comunidades Terapêuticas (Cosentino et al., 2017; Lemes et al., 2020; Danieli, Ferreira, Nogueira, Oliveira, Cruz e Araujo Filho, 2017). O predomínio de indivíduos solteiros, revela uma dificuldade desses usuários manterem-se em relacionamentos, certamente ocasionado pela dependência química, uma vez 
que o indivíduo reduz o seu tempo dedicado ao envolvimento amoroso, voltando-se para o uso de drogas psicoativas (Silva e Tiyo, 2017). Outro ponto a ser considerado é o fato da dependência se tornar algo penoso para as pessoas próximas e familiares dificultando assim o envolvimento ou permanência em relacionamentos estáveis (Santos, Carvalho e Miranda, 2014).

A distribuição dos internos segundo a cor da pele demonstrou participantes que se autodeclararam pardos; dados similares foram encontrados em outros dois estudos realizados no interior do Centro-Oeste (Lemes et al., 2020; Nascimento et al., 2019). Já em Minas Gerais, a população encontrada predominou usuários negros (Madalena \& Sartes, 2018).

Apesar de estarem em idade produtiva para o mercado de trabalho, os usuários deste estudo não possuíam renda mensal. A ausência de renda também foi apontada em outros estudos realizados com pessoas nessa mesma situação (Cosentino et al., 2017; Lemes et al., 2020), indicando que pessoas usuárias de drogas apresentam dificuldade em se manterem no mercado de trabalho, seja formal ou informal, e desta forma não possuem atividade econômica remunerada. Entretanto um fator que corrobora com o desemprego é a instabilidade no padrão de vida, dada a vulnerabilidade que a dependência química condiciona (Sezorte e Silva, 2019).

Neste estudo os usuários apresentaram baixo nível escolar. O que pode estar relacionado ao uso precoce de SPA, levando ao abandono dos estudos na adolescência (Almeida, Luis, 2017). Assim como essa relação entre a evasão escolar e o uso de substâncias psicoativas também pode ocorrer devido às consequências do consumo ao usuário, como dificuldades cognitivas de percepção, memória e pensamento, acarretando em deficiência de aprendizagem, baixo rendimento e consequente evasão escolar (Almeida, Anjos e Vianna, 2014).

Existe uma correlação entre o fator empregabilidade e a baixa escolaridade, que leva a uma carência na qualificação para atuar no mercado de trabalho, o que pode agravar a dependência química devido à vulnerabilidade social e levar a delinquência, uma vez que o indivíduo necessita de dinheiro para o sustento do consumo (Cosentino et al., 2017).

Os resultados também apontam para a predominância de internos que se autodeclararam protestantes/evangélicos. Dados semelhantes foram verificados em um estudo mineiro (BR), onde a maioria dos entrevistados se autodeclararam protestantes (Madalena e Sartes, 2018), enquanto que em um estudo realizado no interior Paulista (BR) predominou usuários católicos (Danieli et al., 2017). Destaca-se que as CT participantes deste estudo, são dirigidas por instituições religiosas, tal situação pode ter influenciado na autodeclaração do protestantismo pelos usuários, tendo em vista que as organizações religiosas acreditam que a fé é o principal componente no tratamento para a dependência química.

Observou-se neste estudo que o uso de substâncias psicoativas foi marcado pelo início do consumo entre 9 e 20 anos, com um predomínio entre 12 e 17 anos. O uso de substâncias psicoativas tem sido descrita na literatura cada vez mais precoce, com início na adolescência, que é uma fase marcada por diversas mudanças biopsicossociais, o que deixa o indivíduo nessa etapa da vida, mais propenso a comportamentos de risco, elevando o risco deste ter seu primeiro contato com as drogas (Santos et al., 2014). Estudo refere que o uso experimental de drogas em idade precoce, como infância e puberdade, pode ser correlacionado ao uso abusivo e dependência na vida adulta (Cosentino et al., 2017).

Pode-se perceber que as drogas lícitas foram às drogas de entrada entre os participantes do presente estudo, com destaque para o uso de álcool (65\%), semelhante ao que foi verificado em outro estudo (Almeida e Luis, 2017; Fernandes, Ribeiro e Britto, 2018).

Como visto, o uso do álcool foi apontado neste e em outros estudos, como a substância mais consumida entre as pessoas, isso deve-se ao fato de que o álcool tem sido apontado como a droga mais consumida em todo o mundo (Fernandes et al., 2018), assim como entre os brasileiros, como apontou o III Levantamento Nacional sobre o uso de Drogas pela população Brasileira (Bastos, Vasconcellos, Boni, Reis \& Coutinho, 2019).

Além disso, destaca-se que o álcool é uma substancia lícita, ou seja, socialmente aceita e facilmente comercializada, 
embora seja proibido a sua distribuição e uso entre menores de idade (Gusmão et al., 2017). No entanto, seu consumo em excesso pode causar sérios prejuízos à saúde física e mental dos consumidores, além de predispor ao uso de substâncias ilícitas (Santos et al., 2014).

Além do álcool ter sido a droga de preferência para iniciar o consumo entre os participantes deste estudo, ela também foi a droga que, na percepção dos usuários, contribuiu para a condição de dependência, seguido pelo crack. Outros estudos com mesmo delineamento também encontraram uma forte prevalência do uso do álcool (Lemes et al., 2020; Silva et al., 2017), assim como existe uma alta procura pelo tratamento da dependência química por usuários de Crack (Lemes et al., 2020; Madalena \& Sartes, 2018).

Um estudo observou que o consumo abusivo de álcool antecede ao uso das drogas ilícitas, havendo uma redução nesse consumo após o início do uso de substâncias ilícitas, com exceção ao crack e a cocaína, nesse caso o abuso de álcool é consequente ao uso dessas drogas, devido sua capacidade de atenuar os efeitos negativos e estimular o efeito da euforia causado por elas, servindo também como um gatilho para o uso (Almeida \& Luis, 2017).

$\mathrm{O}$ uso de SPA foi iniciado entre os participantes deste estudo precocemente, consumido por motivos de curiosidades e influências de amigos. A influência de amigos também pode ser verificada em outro estudo que apontou essa influência entre 63\% dos participantes, como um dos principais motivos para o consumo (Santos et al., 2014). Destaca-se ainda que é comum observar adolescentes sendo influenciado por amigos e colegas, inclusive quando se diz respeito ao uso de drogas. Além do mais, a curiosidade em viver novas experiências e sensações, são os principais fatores que os levam a se aproximarem das drogas (Santos et al., 2014).

Nesta pesquisa foi expressivo o número de participantes que reportaram o histórico familiar entre parente de primeiro grau de consumo de SPA, assim como também foi mencionado em um estudo realizado em uma Comunidade Terapêutica em São Paulo (BR), onde, 70\% dos casos reportaram o consumo de drogas entre familiares de primeiro grau (Danieli et al., 2017). Considera-se um fator de risco para o abuso de substâncias psicoativas, a existência do convívio direto com outras pessoas que também consomem álcool de forma nociva. É importante ressaltar que os filhos tendem a repetir o comportamento dos pais e ainda estão suscetíveis pela predisposição genética, que também se constitui um fator de risco para a dependência química (Danieli et al., 2017).

Levando em consideração que a família possui um importante papel fundamental no tratamento da dependência química em conjunto com o próprio usuário, participando do processo de reabilitação, prevenção a recaída, e no desafio de superar o quadro de dependência, neste e em outros estudo, a família se mostrou um fator de risco ao consumo, por apresentarem parentes de primeiro grau também usuários de drogas (Silva \& Tiyo, 2017; Perrone \& Correa, 2019). Por outro lado, a família tem sido considerada como um fator de proteção ao uso de drogas, quando existe uma convivência familiar respeitosa e atenciosa a todos os membros, o que pode favorecer a formação de resiliência diante das situações adversas (Silva \& Tiyo, 2017).

O uso de substâncias psicoativas vem causando uma serie de danos/prejuízos aos usuários, em várias dimensões de sua vida, seja ela física, mental ou social (Cosentino et al., 2017; Lemes et al., 2020; Danieli et al., 2017). O presente estudo destacou a ocorrência de danos ao próprio usuário, seja de ordem física (doenças respiratórias, desnutrição e déficit neurológico) ou mental (depressão e ansiedade). Em contrapartida, a maioria dos participantes negaram ter causado prejuízos a outras pessoas.

Quanto aos danos físico, outros estudos também destacam os prejuízos causados ao sistema respiratório (Madalena \& Sartes, 2018), certamente isso ocorre em decorrência do uso de SPA (Silva et al., 2017), da inalação das substâncias e da fumaça, seja do consumo de cigarro, maconha, crack ou cocaína, elevando o risco do surgimento de doenças como o câncer de pulmão, enfisema, tuberculose, etc. 
A desnutrição foi outro comprometimento físico reportado entre os entrevistados deste estudo, pode estar relacionada à situação de vulnerabilidade que o indivíduo se encontra, como o desemprego e a mudança nos padrões de alimentação. Além disso, o uso de drogas estimulantes, como o crack e a cocaína, compromete o sistema que regula a fome, prejudicando o bem-estar do usuário, levando-o a desnutrição (Silva et al., 2016).

Os participantes desse estudo também revelaram um déficit neurológico, causado em decorrência do consumo de droga, como dificuldade de atenção, concentração, aprendizado, assim como o relatado em um estudo realizado com familiares de usuários de drogas (Cosentino et al., 2017). O comprometimento neurológico pode contribuir para a baixa escolarização do usuário, bem como a dificuldade de permanência em vínculos afetivos, ou em manter empregos remunerados, resultados esses verificados no presente estudo.

Com relação a saúde mental, este estudo destacou segundo os participantes que a dependência química contribuiu para o surgimento da depressão e ansiedade, o que também foi reportado em outro estudo realizado com usuários de SPA (Silva et al., 2016). Parte dos entrevistados do presente estudo refere já ter tentado suicídio alguma vez na vida, o que também foi mencionado entre 16,7\% dos usuários de um estudo Mineiro (BR) (Madalena e Sartes, 2018). Os dados destacam a importância de um acompanhamento psicológico e psiquiátrico durante o tratamento para dependência química em CT, a fim de que se ofereça um cuidado holístico que respeite as necessidades dos internos e ainda contribua para uma melhor adesão ao tratamento (Madalena \& Sartes, 2018).

Outro aspecto avaliado neste estudo é a ocorrência do consumo de múltiplas drogas anterior ao inicio do tratamento, como o álcool, tabaco, crack e a maconha. O poliuso de drogas também foi verificado em outras pesquisas (Danieli et al., 2017; Santos et al., 2014). O uso concomitante de diversas drogas é comum entre pessoas que se encontram em situação de dependência química, o que pode favorecer a presença de conduta agressiva e criminal, comorbidades psiquiátricas como a depressão e até mesmo o comportamento sexual de risco e outros agravos associados (Santos et al., 2014).

Estudos apontam que o álcool tem sido comumente consumido com o crack, o que pode estar relacionado ao fato de que o álcool é uma droga depressora do Sistema Nervoso Central (SNC), amenizando os sintomas de inquietação psicomotora e ansiedade, que comumente aparece após o uso do crack (Santos et al., 2014; Fernandes et al., 2018).

Desde a antiguidade, a bebida alcoólica sempre foi a droga de maior difusão entres os povos, esse consumo é inerente a fatores culturais, se fazendo presente em festas e comemorações e em determinados rituais religiosos. Um estudo reportou que o álcool tem contribuído como porta de entrada para o consumo de outras substâncias (Madalena \& Sartes, 2018). Dados apontam que mesmo com o aumento da variabilidade de drogas devido ao avanço do tráfico (Perrone \& Correa, 2019), o álcool continua sendo a droga de maior consumo, por ser lícita.

Neste estudo, verificou que a maioria dos entrevistados consumiam SPA diariamente, com frequência de duas a dez vezes ou mais ao dia, anterior ao tratamento atual na CT, comprometendo assim sua rotina diária, trazendo prejuízos em todas as esferas (pessoal, familiar, social) e caracterizando como dependência química. Esse uso diário de drogas também foi apontado por participantes de um estudo realizado em um CAPS AD II no Rio Grande do Norte (BR) (Santos et al., 2017). As principais consequências do uso cotidiano de drogas é o prejuízo a saúde física e mental, como já mencionados neste estudo, além de desenvolver problemas sociais graves, como detenção, e crônicos, como incapacidade em relação ao trabalho (Diagnostic and Statistical Manual of Mental Disorders, 2014).

Prevaleceu neste estudo indivíduos que buscaram pelo tratamento de forma voluntária e através de familiares, dado semelhante foi verificado em outra pesquisa que também avaliou usuários de comunidades terapêuticas (Madalena e Sartes, 2018), evidenciando uma ausência de internações compulsória. Um estudo Mato-grossense (BR) revelou uma forte associação entre aderir ao tratamento em comunidade terapêutica de forma voluntaria, o fato de considerar o tratamento recebido adequado e a diminuição de vontade de usar drogas no tratamento atual, fatores que podem favorecer a adesão ao 
tratamento (Lemes et al., 2020).

Em relação ao número de internações para tratar a dependência química, mais da metade dos entrevistados, reportaram três ou mais internações pregressas. Múltiplas internações também foram reportadas em outros estudos (Danieli el at., 2017; Fernandes et al., 2018). Os dados apontam uma alta reincidência no tratamento para a dependência química, o que pode estar relacionado a fatores intrapessoal e interpessoal da recaída percebida pelo usuário, apontando a autoeficácia, expectativa do resultado, estágio motivacional, dificuldade de enfrentar os problemas da vida diária, estado emocional e fissura, como sendo os determinantes da recaída. Por isso, deve-se levar em consideração que a recaída é um processo complexo e dinâmico, e que os fatores que corroboram para a dependência química ocorrem simultaneamente, apontando a dificuldade em considerar todos esses aspectos na hora de desenvolver o tratamento (Ferreira et al., 2016).

\section{Considerações Finais}

Este estudo revelou o perfil de usuários de substâncias psicoativas residentes em comunidades terapêuticas, localizadas no interior do Vale do Araguaia (BR), destacando um consumo entre homens adultos jovens, solteiros, sem renda, com pouca escolaridade, com início do consumo de drogas precoce, tendo as drogas licitas como drogas de entrada, prevalência do poliuso, histórico familiar de dependência química e alta reincidência no tratamento, com comprometimento em sua saúde física e mental.

Os resultados deste trabalho podem favorecer a discussão dos principais aspectos envolvendo a dependência química e contribuir para o planejamento da assistência em saúde e/ou social prestada a esse perfil da população.

Além disso, salienta-se a importância de que sejam desenvolvidas estratégias preventivas precoces, principalmente direcionadas a adolescentes, priorizando o grupo de risco, assim como o suporte a usuários antes de se encontrar em situação de dependência, evitando assim, prejuízos na fase adulta.

Outro ponto relevante, é que a discussão das características desses indivíduos, pode favorecer o aperfeiçoamento da assistência de profissionais de saúde, em especial os da enfermagem, já que esses profissionais se encontram mais presentes nos ambientes de cuidado destinado a essa população, em qualquer modalidade de atendimento que este preferir buscar para se tratar. Uma vez que os enfermeiros têm sido os responsáveis por planejar, manejar e atender as demandas dessa população, respeitando a autonomia de sua profissão, podendo ainda, contribuir para a adesão ao tratamento desses usuários.

Destaca-se que esse estudo atingiu seu objetivo, dentro de suas limitações, por se tratar de uma pesquisa realizada com uma amostra reduzida, apesar de não se ter a intenção de generalizar os resultados. Com isso, a partir da análise de seus resultados, pode se somar a literatura já existente, ampliando o conhecimento sobre essa população.

Novas pesquisas sobre a temática, com um maior número de usuários, e de ambos os sexos, são necessárias para abranger o conhecimento sobre o perfil sociodemografico e de consumo de substâncias, uma vez que essa compreensão é um fator importante para que ações preventivas sejam realizadas com maior efetividade.

\section{Referências}

Almeida, C. S., \& Luis, M. A. V. (2017). Caracteristicas sociodemograficas e padrão de uso de crack e outras drogas em um CAPS AD. Revista de enfermagem UFPE on line, 11(Supl. 4):1716-23.

Almeida, R. A. A., Anjos, U. U., Vianna, R. P. T., \& Pequeno, G. A. (2017). Perfil dos usuários de substâncias psicoativas de João Pessoa. Saúde Debate, $38(102), 526-538$.

Bastos, F. I. P. M., Vasconcellos, M. T. L. D., Boni, R. B., Reis, N. B. D., \& Coutinho, C. F. D. S. (2017). III levantamento nacional sobre o uso de drogas pela população brasileira: FIOCRUZ/ICICT, 528 p.

Bastos, F. I. P. M., Vasconcellos, M. T. L. D., De Boni, R. B., Reis, N. B. D., \& Coutinho, C. F. D. S. (2019). III Levantamento Nacional sobre uso de Drogas pela População Brasileira Documentação Complementar II - Versão Janeiro de 2019. MS/ FIOCRUZ.

Bearn, J., \& O'Brien, M. (2015). "Addicted to Euphoria": The History, Clinical Presentation, and Management of Party Drug Misuse. International review of neurobiology, 120, 205-233. 
Research, Society and Development, v. 10, n. 1, e22110111677, 2021

(CC BY 4.0) | ISSN 2525-3409 | DOI: http://dx.doi.org/10.33448/rsd-v10i1.11677

Capistrano, F. C., Maftum, G. J., Mantovani, M. F., Felix, J. V. C., Kalinke, L. P., Nimtz, M. A., \& Maftum, M. A. (2018). Consequências do uso abusivo de substâncias psicoativas por pessoas em tratamento. Revista Saúde e Pesquisa, 11(1), 17-26.

Cosentino, S. F., Vianna, L. A. C., Souza, M. H. N., \& Perdonssini, L. G. B. (2017). Características de cuidadores familiares e de usuários de drogas. Revista de enfermagem UFPE on line, 11(6), 2400-7.

Danieli, R. V., Ferreira, M. B. M., Nogueira, J. M., Oliveira, L. N. C., Cruz, E. M. T. N., \& Araujo, G. M., Filho. (2017). Perfil Sociodemográfico e Comorbidades Psiquiátricas em Dependentes Químicos acompanhados em Comunidades Terapêuticas. Jornal brasileiro de psiquiatria, 66(3).

Diagnostic and Statistical Manual of Mental Disorders. (2014). Manual diagnóstico e estatístico de transtornos mentais: DSM-5. Artmed, (5a ed.)

Fernandes, M. A., Ribeiro, M. M. M., \& Britto, L. B. (2018). Caracterização de dependentes químicos em tratamento em uma comunidade terapêutica. Revista de enfermagem UFPE on line, 12(6), 1610-7.

Ferreira, A. C. Z., Czarnobay, J., Borba, L. O., Capistrano, F. C., Kalinke, L. P., \& Maftum, M. A. (2016). Determinantes intra e interpessoais da recaída de dependentes químicos. Revista Eletronica de Enfermagem, 18.

Gil, A. C. (2008). Como elaborar projetos de pesquisa: Atlas. (4a ed.)

Gusmão, P. P., Fernandes, R. F. D., Rezende, R. C., Bonfim, R. S., Porto, Y. V., Fernandes, L. C., \& Moura, L. R. (2017). Perfil epidemiológico de uma população de usuários de drogas de Anápolis, Goiás. Revista Educação e Saúde, 5(1), 28-37.

Lemes, A. G., Rocha, E. M., Nascimento, V. F., Silva, L. S., Almeida, M. A. S. O., \& Villar, M. A. L. (2020). Caracterização de usuários de drogas psicoativas residentes em comunidades terapêuticas no Brasil. Enfermería Global, (58), 436.

Lessa, M. B. M. F. (1998). Os paradoxos da existência na história do uso das drogas.

Ministério da Cidadania. Secretaria Nacional de Cuidados e Prevenção às Drogas. Curso COMPACTA: capacitação de monitores e profissionais das comunidades terapêuticas/Ministério da Cidadania, Secretaria Nacional de Cuidados e Prevenção às Drogas: SEAD/UFSC, 386p.

Madalena, T. S., \& Sartes, L. M. A. (2018). Usuários de crack em tratamento em Comunidades Terapêuticas: perfil e prevalência. Arquivos Brasileiros de Psicologia, 70 (1), 21-36.

Nascimento, V. F., Lima, C. A. S., Hattori, T. Y., Terças, A. C. P., Lemes, A. G., \& Luis, M. A. V. (2019). Daily life of women with alcoholic companions and the provided care. Anais da Academia Brasileira de Ciencias, 91: e20180008.

Pereira, A. S., Shitsuka, D. M., Parreira, F. J. \& Shitsuka, R. (2018). Metodologiada pesquisa científica.: UFSM, NTE.

Perrone, P. A. K., \& Correa, F. K. (2019). Fatores associados à recidiva e abandono do tratamento de dependentes químicos: um estudo longitudinal em duas Comunidades Terapêticas. Faculdade de Medicina (FMB) UNESP.

Rouquayrol, Z. M. (1994). Epidemiologia \& Saúde. Rio de Janeiro: Medsi Editora Médica e Científica Ltda, 527 p.(4a ed.)

Santiago, J. (2001). A droga do toxicômano: uma parceria cínica na era da ciência: Jorge Zahar.

Santos, R. C. A., Carvalho, S. R., \& Miranda, F. A. N. (2014). Perfil socioeconômico e epidemiológico dos usuários do Centro de Atenção Psicossocial Álcool e Drogas II de Parnamirim, RN, Brasil. Revista brasileira de Pesquisa Saúde, 16(1), 105-111.

Sezorte, F. O. F., \& Silva, J. V. (2019). Perfil dos usuários de Substâncias Psicoativas (SPA) acolhidos na Comunidade Terapêutica religiosa Shalom. Revista Valore, 4(Edição Especial), 426-438.

Silva, A. C., \& Tiyo, R. (2017). Perfil de dependentes químicos atendidos na unidade de reabilitação no hospital psiquiátrico de Maringá: uma fonte de pesquisa. Uningá Review, 29(1), 61-63.

Silva, D. L. S., Torrezan, M. B., Costa, J. V., Garcia, A. P. R. F., \& Toledo, V. P. (2017). Perfil sóciodemográfico e epidemiológico dos usuários de um centro de atenção psicossocial álcool e drogas. Revista de Enfermagem Atenção Saúde, 6(1), 67-79.

Silva, E. R., Ferreira, A. C. Z., Borba, L. O., Kalinke, L. P., Nimtz, M. A., \& Maftum, M. A. (2016). Impacto das drogas na saúde física e mental de dependentes químicos. Ciencia, Cuidado e Saúde, 15(1), 101-108.

United Nations Office on Drugs and Crime. World Drug Report. (2020). Relatório Mundial sobre Drogas 2020: consumo global de drogas aumenta, enquanto COVID-19 impacta mercados, aponta relatório. Vienna. 